\title{
Rupture complète du tendon quadricipital
}

\section{Quadriceps tendon rupture}

\section{E. Dazin $\cdot$ M. Planchet $\cdot$ Y. Daniel}

Reçu le 10 janvier 2012 ; accepté le 24 février 2012

(C) SFMU et Springer-Verlag France 2012

Un homme de 53 ans, en surpoids, sans autre antécédent, se plaint aux urgences d'une douleur du genou gauche, après une chute dans les escaliers. L'examen révèle une extension impossible du genou ainsi qu'une dépression au dessus de la rotule. Il présente une rupture complète du tendon quadricipital (Fig. 1).

Le diagnostic est purement clinique. Une solution de continuité est généralement visible et palpable au dessus de la rotule [1], bien que celle-ci puisse parfois être absente, comblée par l'œdème ou l'hématome. La rupture du tendon quadricipital doit être évoquée lors de l'impossibilité d'étendre le genou dans un contexte douloureux avec une flexion conservée.

Cependant la radiographie du genou est nécessaire afin d'évaluer la qualité osseuse de la patella et de rechercher un arrachement osseux. L'échographie montre le niveau de rupture.

L'intervention chirurgicale, devant une rupture complète, est alors nécessaire et ne doit pas être différée [2].

\section{Références}

1. Johnson AE, Rose SD (2006) Bilateral quadriceps tendon ruptures in a healthy, active duty soldier: Case report and review of the literature. Mil Med 171:1251

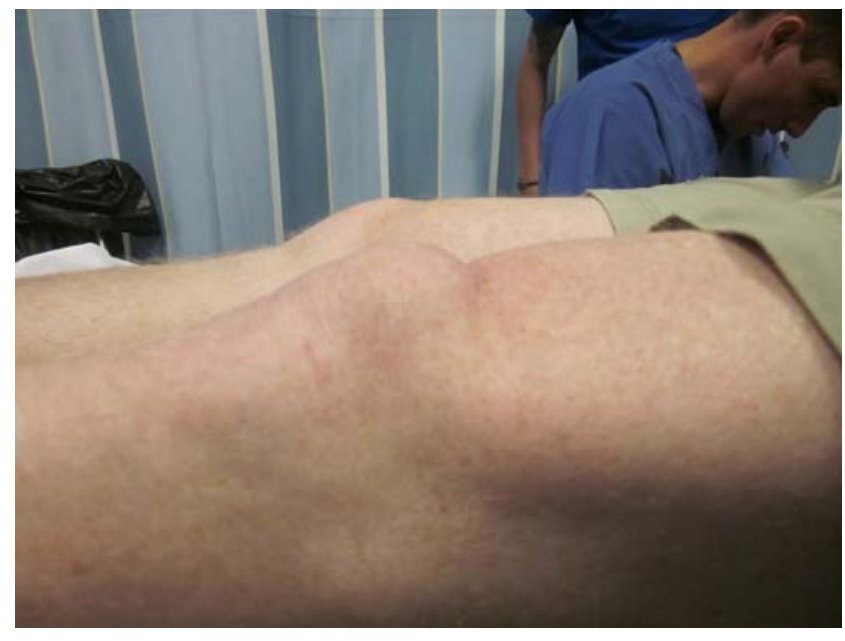

Fig. 1 Rupture complète du tendon quadricipital

2. Boggione C, Graveleau N, Saillant G (2005) Traitement des ruptures du tendon quadricipital. J Traumatol Sport 22:19-25
E. Dazin $(\bowtie) \cdot$ M. Planchet $\cdot$ Y. Daniel

Régiment d'infanterie - Chars de marine, Quartier Ladmirault, Service médical, 46 bis, rue Jean Mermoz, F-86000 Poitiers, France

e-mail : emiliedazin@hotmail.fr 